\title{
Functional Outcome of Displaced Diaphyseal Both Bone Forearm Fracture in Children Treated with Elastic Stable Intramedullary Nails
}

\author{
DC GS
}

\begin{abstract}
Introduction: Pediatric forearm fractures are most commonly managed by closed reduction and above elbow cast. Failure to achieve anatomical initial reduction, tight cast and re-displacement within cast leading to malunion are common complication .Elastic stable intramedullary nailing for such fracture gives more predictable result with minimal morbidity. We studied on functional outcome of such fracture treated operatively with elastic stable intramedullary nail in our center. Methods: This prospective descriptive study was done in Nepalgunj Medical College Hospital, Nepalgunj between January 2015 to May 2017. It included 47 pediatric patients (5-15 years) with displaced both bone forearm diaphyseal fracture. All fractures were fixed with elastic stable intramedullary nails of appropriate size under general anesthesia. Cases were followed up at 1, 2, 4, 6, 8, 10, 12 weeks and at 6 month functional outcome was evaluated using price criteria. Data were entered in structured proforma and statistical analysis was carried out using SPSS 20.0. Results: Out of 47 cases included in our study, mean age of patient was 9.7 years (range 5-15 years). 31(65.9\%) cases were male and $16(34.1 \%)$ were female.42(89.4\%) cases had closed fracture and 5(10.6\%) had grade I open factor.40(85.1\%) cases were operated by closed technique. Average hospital stay was $2.7 \pm 1.8$ day (range 2-5days). Radiological union was achieved on $6.5 \pm 2.7$ weeks (range 6-11weeks). Functional outcome in final follow up at 6 month was excellent in 36(76.6\%), good in 9(19.1\%) and fair in 2(4.3\%) cases. Superficial surgical site infection was seen in 3 cases (6.3\%) and exposure of nail tip was seen in 2 cases (4.2\%). Conclusion: Elastic stable intramedullary nail for displaced pediatric both bone forearm diaphyseal fracture is biological osteosynthesis, simple and reproducible treatment with excellent functional outcome with minimal morbidity.
\end{abstract}

Keywords: Elastic Stable Intramedullary Nail (ESIN), forearm fracture, pediatric

\section{INTRODUCTION}

Pediatric forearm fractures are common orthopedic injuries ${ }^{1}$. Traditionally most of the displaced both bone forearm fracture are managed successfully with closed reduction and above elbow cast application ${ }^{2}$. Malunion(5-15\% cases) with functional disability due to redisplacement within cast is the most common complication of displaced diaphyseal both bone forearm fracture managed by cast application ${ }^{3}$. The most common indications for surgery in pediatric both bone forearm fracture are failure of closed reduction, redisplacement, unstable fracture ${ }^{4.5}$. Adequate stabilization can be achieved by various surgical techniques for these types of fractures including plates, external fixation and elastic stable intramedullary nail $(E S I N)^{6-7.8}$. Over last 10 years approximately one fourth of such fracture are treated by $\mathrm{ESIN}^{9}$. In this study we prospectively followed pediatric (5-15 years) displaced both bone forearm diaphyseal fracture managed by ESIN in our center, evaluated the functional outcome of those cases and noted complications with this treatment method.

Address for correspondence:

Dr. Gopal Sagar DC

Department of Orthopedics

Nepalgunj Medical College and Teaching Hospital

Nepalgunj, Banke

E-mail:sagargopal@gmail.com

\section{METHODS}

This prospective descriptive study was done in department of orthopedics in Nepalgunj Medical College Teaching Hospital, Nepalgunj after appropriate ethical clearance. All Pediatric (5-15 years) diaphyseal both bone forearm fractures (grossly displaced/angulated) presenting to the orthopedic department between January 2015 and May 2017 were included in this study. Metaphyseal both bone forearm fracture, isloated fracture of forearm, pathological fracture, Grade II and III open fractures, refracture cases were excluded from the study. Forty seven cases fulfilled the inclusion criteria and were included in this study. Demographic variables, mode of injury, fracture pattern were noted in proforma.

Cases were taken up for surgery under general anesthesia and were operated by first author. Intravenous antibiotics (Cefazolin, 500mg) stat dose was given to all cases. First closed reduction under c-arm was attempted in all cases after standard sterile draping and antiseptic painting, if closed reduction fails then fracture site was opened with small incision centering fracture and reduction was achieved. Fracture were fixed sequentially first radius and then ulna with ESIN of appropriate size (nail that fills $80 \%$ of diameter of the bone was chosen, usually 2 or $2.5 \mathrm{~mm}$ ). Entry portal for radius was made by mini incision over dorsolateral aspect of distal radius sparing physis and nail was introduced in retrograde fashion. Entry portal for ulna was made over dorsomedial aspect of proximal metaphyseal region and nail was introduced in antegrade fashion under C-arm control. Wound was closed 
with skin stapler and dressing was applied. Crepe bandage compression with arm pouch sling support was applied in all case. We didn't applied any slab or cast for immobilization. Cases were usually discharged after $48 \mathrm{hrs}$. of observation for any early fracture complications specially compartment syndrome.

Cases were followed up in 1, 2, 6, 8, 10 and 12 weeks and 6 months after surgery. At 2 weeks stapler was removed and gentle range of movement out of sling was started. Check $x$-ray was done at 6,8 weeks if no radiological union is seen then $x$ ray are repeated at 10 and 12 weeks sequentially to look for radiological union and same was noted. At 6 month follow-up functional outcome was evaluated as per price et a ${ }^{10}$ criteria and was recorded as excellent, good, fair and poor. Data were then entered in Microsoft excel and analyzed for frequency distribution and mean where appropriate, using SPSS 20.0 version.

\section{RESULTS}

In our study out of 47 cases of displaced diaphyseal both bone forearm fracture managed by ESIN, 31(61.9\%) cases were male and $16(34.1 \%)$ were female. Mean age of patient was 9.7 years.

Mode of injury was fall on outstretched hand while playing outdoor in $27(57.4 \%)$ cases, slip injury while walking in $12(25.5 \%)$ cases and road traffic accident in $8(17.1 \%)$ cases. Right forearm was fractured in 28 cases, left forearm was fractured in 19 cases.

Both bone fracture was a mid-diaphysis in $29(61.7 \%)$ cases, distal diaphysis in $10(21.2 \%)$ cases and proximal diaphysis in $8(17.1 \%)$ cases. Of them $42(89.4 \%)$ cases were closed fracture and $5(10.6 \%)$ cases were grade I open fracture.

Of them anatomical reduction and fixation with ESIN was done by closed reduction technique in $40(85.1 \%)$ cases. However 7 (14.9\%) cases required open reduction, of them 3 cases required open reduction only for radius fracture and 4 cases required open reduction for both radius and ulna. Average length of hospital stay in these cases were $2.7 \pm 1.8$ days (ranging 2-5 days), longer stay was required for grade I open fracture case and those who required open reduction for fixation.

On follow-up x-ray radiological union was achieved on means duration of $6.5 \pm 2.7$ weeks (6-11weeks). (Figure 1). ESIN removal was done in all patient after 7.2 \pm 2.3 months (6-12 months)
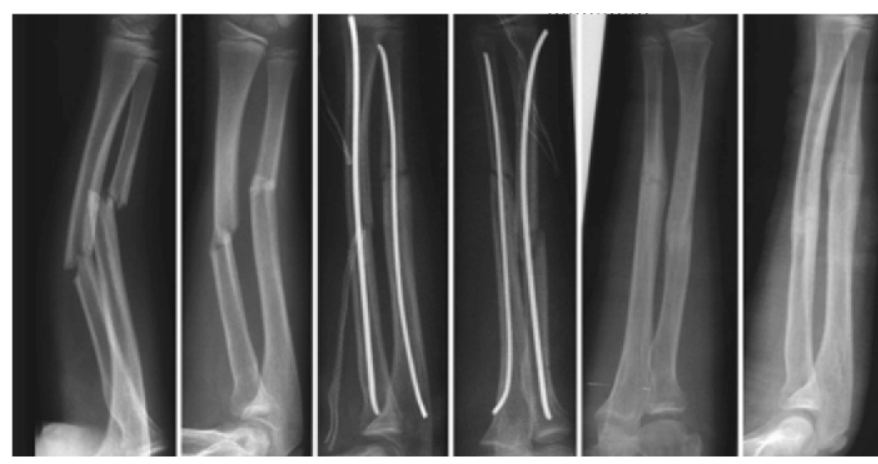

Figure.1: X-ray showing displaced both bone forearm diaphyseal fracture in 10 year old child. Immediate post-op and at 8 weeks follow-up x-ray with radiological union.

Functional outcome evaluated by price criteria showed excellent result in about three fourth of the cases. (Figure.2)

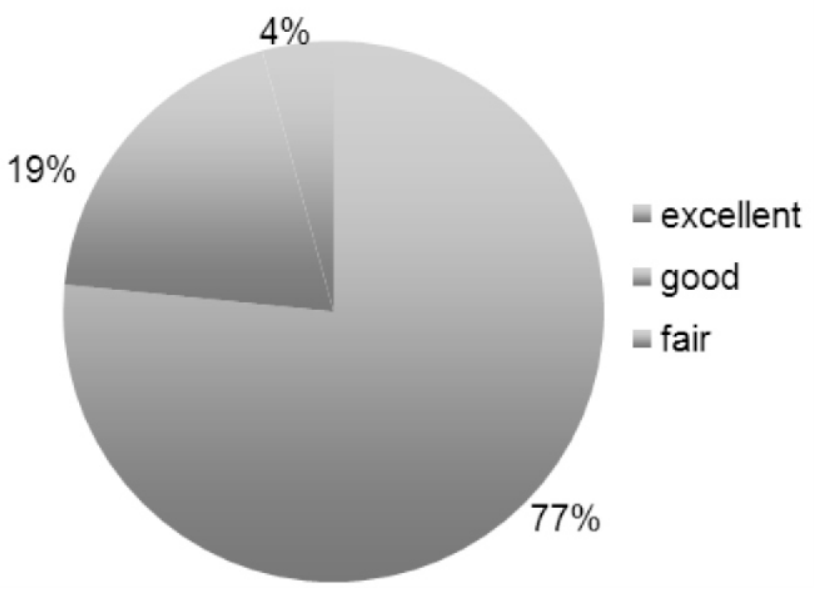

Figure 2: Functional outcome evaluated by price et al criteria

Post-operative complications noted were superficial surgical site infection in 3 cases (6.3\%) managed with oral antibiotics and did not progress to deep infection or osteomyelitis. Exposure of nail tip over ulnar insertion site was seen in 2 cases $(4.2 \%)$ and was managed with regular dressing and early nail removal after radiological union.

\section{DISCUSSION}

Closed reduction and casting is most common method of treating pediatric diaphyseal forearm fractures, surgery is done only for unstable fracture or when closed reduction fails or there is redisplacment within cast ${ }^{11}$. There is high incidence of malunion in such conservatively treated fracture as diaphyseal fracture has less remodeling potential then distal one third forearm fracture ${ }^{1}$. Daruwalla JS suggested surgery for midshaft and proximal forearm fractures with angulations $>10^{\circ}$ because of limited remodeling potential in these areas of the bone $^{4}$. Given the potential failure of non-operative management (1.5\% to $31 \%)$ and the importance of minimizing 
angular deformity to preserve normal forearm rotation, operative management of pediatric forearm fracture has been increasingly popular ${ }^{13}$. Shoemaker et al suggested that the ideal mode of fixation of pediatric forearm fractures should maintain alignment, be minimally invasive and inexpensive, and carry an acceptable risk profile ${ }^{8}$. The main advantages of intramedullary nailing include maintenance of reduction, provision of an inexpensive, less invasive, relatively easy application, protection of bone alignment by three point contact, acceleration of bridging callus formation through micro movements at the fracture site, and thus contribution to rapid bony healing ${ }^{14}$.

In our study anatomical reduction and fixation with ESIN was done by closed reduction technique in $40(85.1 \%)$ cases. However $7(14.9 \%)$ cases required open reduction, of them 3 cases required open reduction only for radius fracture and 4 cases required open reduction for both radius and ulna. The rate of open reduction with intramedullary nailing of pediatric forearm fracture in published literature ranges from $7.4 \%$ to $75 \%^{15,16}$. Close reduction or open reduction before intramedullary nailing yield similar functional results, with similar complication profile in pediatric diaphyseal fracture ${ }^{13}$.

In our study radiological union was achieved in all cases in mean duration of $6.5 \pm 2.7$ weeks (6-11weeks). There was no case of malunion or nonunion in our study. Shivanna $\mathrm{S}$ et al reported radiological union was achieved in all their patients at an average of 7 weeks and similar to our study they did not report any case of delayed union, malunion or nonunion.

In our study Functional outcome evaluated at 6 month follow up by price et al criteria showed excellent result in 36(76.6\%), good in $9(19.1 \%)$ fair in $2(4.3 \%)$ cases and none had poor results. Ozkaya U and Parmaksizoðlu AS recorded that $85.7 \%$ had excellent, $14.3 \%$ had good results according to Price et al Criteria18. Richter et al observed only three of 30 patients with a supination deficit of $10^{\circ}$ after an average of 6 months followup i.e. $90 \%$ excellent results ${ }^{19}$.

In our study post-operative complications noted were superficial surgical site infection in $3(6.3 \%)$ cases, which was managed successfully with oral antibiotics and did not progress to deep infection or osteomyelitis. Exposure of nail tip over ulnar insertion site was seen in $2(4.2 \%)$ cases. Yalcinkaya $\mathrm{M}$ et $\mathrm{al}^{2}$ reported complications rate ranged from $4-38 \%$ in patients treated with intramedullary nailing and Flynn JM et al showed that the overall complication rate in patients undergoing intramedullary nailing was $14.6 \%^{13}$.

\section{CONCLUSION}

Closed reduction and casting for unstable pediatric diaphyseal forearm fracture has significant risk of malunion with poor functional outcome.ESIN is safe and reliable biological osteosynthesis for displaced unstable diaphyseal both bone forearm fracture in children. It gives excellent functional outcome with minimal morbidity.

\section{REFERENCES}

1. Amstrong PF, Jouglin VE, Clarke HM, Greene NE, Swiontkowski MF. Pediatric fracture of forearm, wrist and hand. In Skeletal trauma in children, Philadelphia, Saunders 1998; 161- 257.

2. Yalcinkaya M, Dogan A, Ozkaya V, Sokucu S, Uzumcugil O, Kabukcuoglu Y.Clinical results of intramedullary nailing following closed or mini open reduction in pediatric unstable diaphyseal forearm fractures. Acta Orthop Traumatol Turc. 2010;44(1):7-13.

3. Bochang C, Katz K, Weigl D, Jie Y, Zhigang W, Bar-On E. Are frequent radiographs necessary in the management of closed forearm fractures in children? J Child Orthopaed. 2008; 2:217-20.

4. Daruwalla JS. A study of radioulnar movements following fractures of the forearm in children. Clin Orthop Relat Res. 1979; 139:114-20.

5. Fuller DJ, McCullough CJ. Malunited fracture of the forearm in children. J Bone Joint Surg Br. 1982; 64:364-7.

6. Schranz PJ, Gultekin C, Colton CL. Exteranl fixation of fracture in children. Injury. 1982; 23:80-2.

7. Lascombes P, Prevot J, Ligier JN, Metaizeau JP, Poncelet T. Elastic stable intramedullary nailing in forearm shaft fractures in children: 85 cases. J Pediatr Orthop. 1990; 10:167-71.

8. Shoemaker S. Comstock C. Mubarak S, Wenger DR, Chambers HG. Intramedullary Kirschner wire fixation of open or unstable forearm fractures in children. J Pediatr Orthop 1999; 19:329-37.

9. Schmittenbecher PP. State-of-the-art treatment of forearm shaft fractures. Injury 2005; 36(suppl 1):A25-34.

10. Price $C T$, Scott DS, Kurzner ME, Flynn JC. Malunited forearm fractures in children. J Pediatr Orthop. 1990; 10:705-12.

11. Wyrsch B, Mencio GA, Green NE. Open reduction and internal fixation of pediatric forearm fractures. J Pediatr Orthop. 1996; 16:644-50.

12. Sinikumpu JJ, Lautamo A, Pokka T, Serlo W. Complications and radiographic outcome of children's both-bone diaphyseal forearm fractures after invasive and non-invasive treatment. Injury. 2013; 44:431-6.

13. Tarr RR, Garfinkel Al, Sarmiento A. The effects of angular and rotational deformities of both bones of the forearm: an in vitro study. J Bone Joint Surg Am 1984; 66:65-70.

14. Yung PS, Lam CY, Ng BK, Lam TP, Cheng JC. Percutaneous transphyseal intramedullary Kirschner wire pinning: a safe and effective procedure for treatment of displaced diaphyseal forearm fracture in children. J Pediatr Orthop. 2004; 24:7-12.

15. Flynn JM, Jones KJ, Garner MR, Goebel J. Eleven years' experience in the operative management of pediatric forearm fractures. J $\mathrm{P}$ e d i a t $\mathrm{r}$ Orthop. $2010 ; 30: 313-9$. 16. Furlan D, Pogorelić Z, Biočić M, Jurić I, Budimir D, Todorić J, et al. Elastic stable intramedullary nailing for pediatric long bone fractures: experience with 175 fractures. Scand J Surg. 2011; 100:208-15.

17. Shivanna S, Maruthi CV. Pediatric forearm fractures with tens: freedom of movements. International Journal of Research in Orthopaedics. 2016 Sep 3;2(3):143-7.

18. Ozkaya U, Parmaksizo lu AS, Kabukçuo lu Y, Yeniocak S, Sökücü S., Surgical management of unstable both- bone forearm fractures in children. Acta Orthop Traumatol Turc 2008; 42(3): 188-192.

19. Richter D, Ostermann PA, Ekkernkamp A, et al. Elastic intramedullary nailing: a minimally invasive concept in the treatment of unstable forearm fractures in children. J Pediatr Orthop 1998; 18:457-61. 